VOL. 56 (1997) [135-148]

\title{
AN ACTION OF THE KLEIN FOUR-GROUP ON THE IRRATIONAL ROTATION $C^{*}$-ALGEBRA
}

\author{
P.J. STACEY
}

\begin{abstract}
Explicit automorphisms of the irrational rotation algebra are constructed which are associated with the two $2 \times 2$ diagonal integer matrices of determinant -1 . The fixed point algebra of the product of these two automorphisms is shown to be isomorphic to the fixed point algebra of the flip.
\end{abstract}

\section{THE $\mathbb{Z}_{2} \times \mathbb{Z}_{2}$ ACTION}

Let $\theta$ be an irrational number with $0<\theta<1$ and let the irrational rotation algebra $A_{\theta}$ be the universal $C^{*}$-algebra generated by two unitaries $U, V$ satisfying $V U=e^{2 \pi i \theta} U V$. This algebra has been the subject of extensive study in recent years. In particular it has been shown, as a consequence of the remarkable results in [8] and [9], that there exists a surjective homomorphism from $\operatorname{Aut}\left(A_{\theta}\right)$, the group of *automorphisms of $A_{\theta}$, onto Aut $\left(K_{1}\left(A_{\theta}\right)\right)$, which can be identified with $G L_{2}(\mathbb{Z})$ via the identification of $K_{1}\left(A_{\theta}\right)$ with $\mathbb{Z}^{2}$. It was shown in [6] and [17] how to construct a partial lifting of the map $\operatorname{Aut}\left(A_{\theta}\right) \rightarrow G L_{2}(\mathbb{Z})$ via an isomorphism from $S L_{2}(\mathbb{Z})$ into Aut $\left(A_{\theta}\right)$. The purpose of the present paper is to describe explicit automorphisms of $A_{\theta}$ arising from the matrices $\left(\begin{array}{cc}1 & 0 \\ 0 & -1\end{array}\right)$ and $\left(\begin{array}{cc}-1 & 0 \\ 0 & 1\end{array}\right)$ and to investigate the associated automorphism arising from the matrix $-I$. The construction is given in terms of the inductive limit description of $A_{\theta}$ developed in [9, Section 5] which is now described.

Let the continued fraction expansion of $\theta$ be $\left[m_{0}, m_{1}, m_{2}, \ldots\right]$, with associated partial quotients $p_{0} / q_{0}, p_{1} / q_{1}, p_{2} / q_{2}, \ldots$, and let the natural numbers $a_{n}, b_{n}, c_{n}, d_{n}$ be defined by

$$
\left(\begin{array}{ll}
a_{n} & b_{n} \\
c_{n} & d_{n}
\end{array}\right)=\left(\begin{array}{cc}
m_{4 n} & 1 \\
1 & 0
\end{array}\right)\left(\begin{array}{cc}
m_{4 n-1} & 1 \\
1 & 0
\end{array}\right)\left(\begin{array}{cc}
m_{4 n-2} & 1 \\
1 & 0
\end{array}\right)\left(\begin{array}{cc}
m_{4 n-3} & 1 \\
1 & 0
\end{array}\right) .
$$

Furthermore, for each $k \in \mathbb{N}$, let $k \times k$ matrices $R_{k}$ and $S_{k}$ with entries in $C\left(S^{1}\right)$ be defined by

$$
R_{k}=\left(\begin{array}{cc}
0 & \text { id } \\
I_{k-1} & 0
\end{array}\right) \quad \text { and } \quad S_{k}=\left(\begin{array}{cc}
0 & 1 \\
I_{k-1} & 0
\end{array}\right)
$$

Received 19th September, 1996

Copyright Clearance Centre, Inc. Serial-fee code: 0004-9729/97 \$A2.00+0.00. 
The construction given in [9] gives $A_{\theta}$ as an inductive limit of a sequence $A_{1} \rightarrow A_{2} \rightarrow$ $A_{3} \rightarrow \ldots$ where $A_{n}=M_{q_{4 n}}\left(C\left(S^{1}\right)\right) \oplus M_{q_{4 n-1}}\left(C\left(S^{1}\right)\right)$ and where the connecting maps $\theta_{n}: A_{n} \rightarrow A_{n+1}$ are specified, for constant $A, B$, by

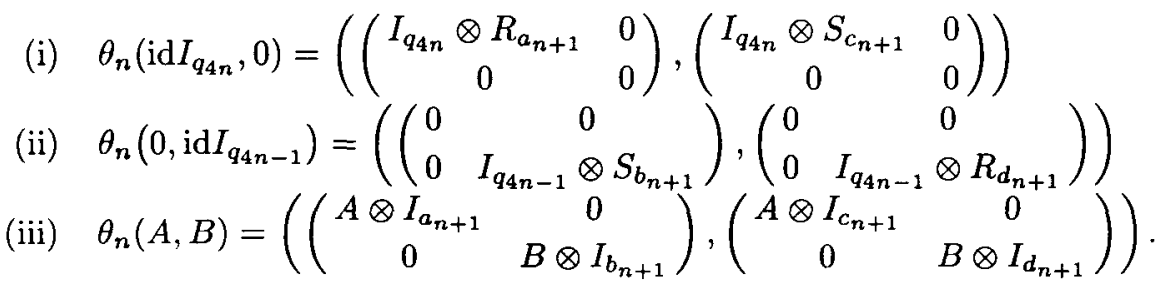

Here, for an $\ell \times \ell$ matrix $M, I_{k} \otimes M$ denotes the $k \ell \times k \ell$ matrix with $k$ copies of $M$ down the main diagonal and $M \otimes I_{k}$ denotes the $k \ell \times k \ell$ matrix consisting of $k \times k$ blocks $m_{i j} I_{k}$ in the obvious way. As the notation indicates, this description corresponds to an isomorphism between $M_{k \ell}$ and the tensor product $M_{k} \otimes M_{\ell}$.

The connecting maps $\theta_{n}$ were chosen to give the following prescribed effect on $K_{0}\left(A_{n}\right)$ and $K_{1}\left(A_{n}\right)$ when these are both identified with $\mathbb{Z}^{2}$ : on $K_{0}\left(A_{n}\right), \theta_{n}$ corresponds to the matrix $\left(\begin{array}{ll}a_{n+1} & b_{n+1} \\ c_{n+1} & d_{n+1}\end{array}\right)$, whereas on $K_{1}\left(A_{n}\right) \theta_{n *}$ corresponds to the identity. It will be important in the sequel to note that the identification of $K_{1}\left(A_{n}\right)$ with $\mathbb{Z}^{2}$ can be given by associating $(1,0)$ with $\left(\left[u_{q_{4 n}}\right],[I]\right)$ and $(0,1)$ with $\left([I],\left[u_{q_{4 n-1}}\right]\right)$, where, for each $k \in \mathbb{N}$,

$$
u_{k}=\left(\begin{array}{cc}
\text { id } & 0 \\
0 & I_{k-1}
\end{array}\right) \text {. }
$$

The inductive limit of the system $A_{1} \rightarrow A_{2} \rightarrow \ldots$ is $A_{\theta}$ because of the main results of [8] and [9], which show that it is sufficient to demonstrate that the inductive limit has the same $K$-theory as $A_{\theta}$.

It may be of interest to note the following alternative way of describing the maps $\theta_{n}$, which will however not be used in the sequel.

Proposition 1.1. Let

$$
\theta_{n}: M_{q_{4 n}}\left(C\left(S^{1}\right)\right) \oplus M_{q_{4 n-1}}\left(C\left(S^{1}\right)\right) \rightarrow M_{q_{4 n+4}}\left(C\left(S^{1}\right)\right) \oplus M_{q_{4 n+3}}\left(C\left(S^{1}\right)\right)
$$

be defined by (i), (ii), (iii) above. Then $\theta_{n}\left(\left(f_{i j}\right),\left(g_{i j}\right)\right)$ is equal to

$$
\left.\left(\begin{array}{cc}
\left(f_{i j}\left(R_{a_{n+1}}\right)\right) & 0 \\
0 & \left(g_{i j}\left(S_{b_{n+1}}\right)\right)
\end{array}\right),\left(\begin{array}{cc}
\left(f_{i j}\left(S_{c_{n+1}}\right)\right) & 0 \\
0 & \left(g_{i j}\left(R_{d_{n+1}}\right)\right)
\end{array}\right)\right)
$$

where, for each $i, j, f_{i j}$ and $g_{i j}$ are elements of $C\left(S^{1}\right)$ and $f_{i j}\left(R_{a_{n+1}}\right), f_{i j}\left(S_{c_{n+1}}\right)$, $g_{i j}\left(S_{b_{n+1}}\right), g_{i j}\left(R_{d_{n+1}}\right)$ use the usual functional calculus for a unitary element of a $C^{*}$-algebra.

PROOF: It is easily seen that the formula given above gives the appropriate answers (i), (ii), (iii) and defines a $*$-homomorphism. 
The $\mathbb{Z}_{2} \times \mathbb{Z}_{2}$ action on $A_{\theta}$ will be defined in terms of certain inductively defined matrices $V_{n}, V_{n}^{\prime}, W_{n}, W_{n}^{\prime}$. To define these, first let $Q_{k}$ be the $k \times k$ matrix

$$
Q_{k}=\left(\begin{array}{lll} 
& & 1 \\
& &
\end{array}\right)
$$

and let $W_{1}=I_{q_{4}}, V_{1}=Q_{q_{3}}, W_{1}^{\prime}=Q_{q_{4}}$ and $V_{1}^{\prime}=I_{q_{3}}$. Then, for each $n \geqslant 1$, let

$$
\begin{aligned}
& W_{n+1}=\left(\begin{array}{ll}
W_{n} \otimes I_{a_{n+1}} & \\
& V_{n} \otimes Q_{b_{n+1}}
\end{array}\right) \\
& V_{n+1}=\left(\begin{array}{ll}
W_{n} \otimes I_{c_{n+1}} & \\
& V_{n} \otimes Q_{d_{n+1}}
\end{array}\right) \\
& W_{n+1}^{\prime}=\left(\begin{array}{ll}
W_{n}^{\prime} \otimes Q_{a_{n+1}} & \\
& V_{n}^{\prime} \otimes I_{b_{n+1}}
\end{array}\right) \\
& V_{n+1}^{\prime}=\left(\begin{array}{ll}
W_{n}^{\prime} \otimes Q_{c_{n+1}} & \\
& V_{n}^{\prime} \otimes I_{d_{n+1}}
\end{array}\right)
\end{aligned}
$$

and, for each $f \in C\left(S^{1}, M_{k}\right)$, where $k \in \mathbb{N}$, let $(R f)(t)=f(\bar{t})$. It will be shown that an action of $\mathbb{Z}_{2} \times \mathbb{Z}_{2}$ on $A_{\theta}$ is defined by the formulae

$$
\begin{aligned}
& \alpha_{n}=\left(A d W_{n},\left(A d V_{n}\right) \circ R\right) \\
& \alpha_{n}^{\prime}=\left(\left(A d W_{n}^{\prime}\right) \circ R, A d V_{n}^{\prime}\right)
\end{aligned}
$$

where $W_{n}, W_{n}^{\prime}, V_{n}, V_{n}^{\prime}$ are regarded as constant matrix valued functions on $S^{1}$.

PROPOSITION 1.2.

(i) $\alpha_{n}, \alpha_{n}^{\prime}$ are automorphisms of $A_{n}$.

(ii) $\alpha_{n}^{2}=$ id, $\alpha_{n}^{\prime 2}=$ id and $\alpha_{n} \alpha_{n}^{\prime}=\alpha_{n}^{\prime} \alpha_{n}$, so that $\alpha_{n}, \alpha_{n}^{\prime}$ define a $\mathbb{Z}_{2} \times \mathbb{Z}_{2}$ action on $A_{n}$.

(iii) $\quad \alpha_{n *}=\left(\right.$ id, - id) and $\alpha_{n *}^{\prime}=(-\mathrm{id}, \mathrm{id})$ on $\mathbb{Z}^{2} \cong K_{1}\left(A_{n}\right)$.

ProOF:

(i) This is clear from the definitions.

(ii) It follows inductively that, for all $n \in \mathbb{N}, W_{n}^{2}=I, W_{n}^{2}=I, V_{n}^{2}=$ $I, V_{n}^{\prime 2}=I, W_{n} W_{n}^{\prime}=W_{n}^{\prime} W_{n}$ and $V_{n} V_{n}^{\prime}=V_{n}^{\prime} V_{n}$, from which the result follows.

(iii) This follows from $R(u)=u^{*}$, where ([I], [u]) and $([\mathrm{u}],[\mathrm{I}])$ are the generators of $K_{1}\left(A_{n}\right)$ described earlier. 
TheOREM 1.1. There exists an isomorphism $\beta$ from $\mathbb{Z}_{2} \times \mathbb{Z}_{2}$ to $\operatorname{Aut}\left(A_{\theta}\right)$ with $\beta(1,0)_{*}=(\mathrm{id},-\mathrm{id})$ and $\beta(0,1)_{*}=(-\mathrm{id}, \mathrm{id})$ on $\mathbb{Z}^{2} \cong K_{1}\left(A_{\theta}\right)$.

Proof: The result will follow from Proposition 1.2 and the fact that $\theta_{n *}=$ id on $K_{1}\left(A_{\theta}\right)$ if it can be shown that $\alpha_{n+1} \theta_{n}=\theta_{n} \alpha_{n}$ and $\alpha_{n+1}^{\prime} \theta_{n}=\theta_{n} \alpha_{n}^{\prime}$ for each $n \in \mathbb{N}$. However

$$
\begin{aligned}
\alpha_{n+1} \theta_{n}\left(\mathrm{id} I_{q_{4 n}}, 0\right) & =\left(\left(\begin{array}{cc}
W_{n} I W_{n}^{*} \otimes R_{a_{n+1}} & 0 \\
0 & 0
\end{array}\right),\left(\begin{array}{cc}
W_{n} I W_{n}^{*} \otimes S_{c_{n+1}} & 0 \\
0 & 0
\end{array}\right)\right) \\
& =\theta_{n}\left(\operatorname{id} I_{q_{4 n}}, 0\right) \\
& =\theta_{n} \alpha_{n}\left(\operatorname{id} I_{q_{4 n}}, 0\right), \\
\alpha_{n+1} \theta_{n}\left(0, \operatorname{id~} I_{q_{4 n-1}}\right) & =\left(\left(\begin{array}{ll}
0 & 0 \\
0 & I \otimes Q_{b_{n+1}} S_{b_{n+1}} Q_{b_{n+1}}
\end{array}\right),\left(\begin{array}{cc}
0 & 0 \\
0 & I \otimes Q_{d_{n+1}} \bar{R}_{d_{n+1}} Q_{d_{n+1}}
\end{array}\right)\right) \\
& =\left(\left(\begin{array}{ll}
0 & 0 \\
0 & I \otimes S_{b_{n+1}}^{*}
\end{array}\right),\left(\begin{array}{cc}
0 & 0 \\
0 & I \otimes R_{d_{n+1}}^{*}
\end{array}\right)\right) \\
& =\theta_{n}\left(\begin{array}{ll}
0,\left(\operatorname{id} I_{q_{4 n-1}}\right)^{*}
\end{array}\right) \\
& =\theta_{n} \alpha_{n}\left(0, \operatorname{id} I_{q_{4 n-1}}\right)
\end{aligned}
$$

and

$$
\begin{aligned}
\alpha_{n+1} \theta_{n}(A, B) & =\left(\begin{array}{cc}
W_{n} A W_{n}^{*} \otimes I_{a_{n+1}} & 0 \\
0 & V_{n} B V_{n}^{*} \otimes I_{b_{n+1}}
\end{array}\right) \\
& \left.\left(\begin{array}{ccc}
W_{n} A W_{n}^{*} \otimes I_{c_{n+1}} & 0 \\
0 & V_{n} B V_{n}^{*} \otimes I_{d_{n+1}}
\end{array}\right)\right) \\
& =\theta_{n}\left(W_{n} A W_{n}^{*}, V_{n} B V_{n}^{*}\right) \\
& =\theta_{n} \alpha_{n}(A, B) .
\end{aligned}
$$

Hence $\alpha_{n+1} \theta_{n}=\theta_{n} \alpha_{n}$. The proof for $\alpha_{n+1}^{\prime} \theta_{n}=\theta_{n} \alpha_{n}^{\prime}$ is exactly similar.

\section{A FIXED POINT ALGEBRA}

It is natural to ask if the $\mathbb{Z}_{2} \times \mathbb{Z}_{2}$ action and the $S L_{2}(\mathbb{Z})$ action on $A_{\theta}$ can be combined to give a $G L_{2}(\mathbb{Z})$ action. If this were to be possible then $\beta(1,1)$ would be conjugate to the flip automorphism, studied in $[\mathbf{3}, \mathbf{4}, \mathbf{5}]$, which is the automorphism of $A_{\theta}$ corresponding to $-I \in S L_{2}(\mathbb{Z})$ under the correspondence established in [6] and [17]. Consequently the fixed point algebra of $\beta(1,1)$ would be isomorphic to the fixed point algebra of the flip. We shall now show this to be the case, which leaves open the possibility of a $G L_{2}(\mathbb{Z})$ action on $A_{\theta}$.

To facilitate the statement of the results let

$$
E_{n}=\left(I_{q_{4 n}}+W_{n} W_{n}^{\prime}\right) / 2 \text { and } F_{n}=\left(I_{q_{4 n-1}}+V_{n} V_{n}^{\prime}\right) / 2
$$


Then $E_{n}$ and $F_{n}$ are the projections associated with the symmetries $W_{n} W_{n}^{\prime}$ and $V_{n} V_{n}^{\prime}$. It will also be convenient to abbreviate $\alpha \alpha^{\prime}=\beta(1,1)$ to $\beta$ and to use $\beta_{n}$ for its restriction to $A_{n}$.

\section{PROPOSITION 2.1.}

(i) The fixed point algebra $B_{n}$ of $\beta_{n}$ is $B_{n}^{1} \oplus B_{n}^{2}$ where $B_{n}^{1}$ is isomorphic to

$$
\left\{f \in C\left([0,1], M_{q_{4 n}}\right): f(0), f(1) \in\left\{E_{n}\right\}^{\prime}\right\}
$$

and $B_{n}^{2}$ is isomorphic to

$$
\left\{f \in C\left([0,1], M_{q_{4 n-1}}\right): f(0), f(1) \in\left\{F_{n}\right\}^{\prime}\right\} .
$$

(ii) $K_{1}\left(B_{n}\right)=\{0\}$.

(iii) The ordered group $K_{0}\left(B_{n}\right)$ is isomorphic to $\mathbb{Z}^{6}$ with positive cone $\left\{\left(n_{1}, n_{2}, n_{3}, n_{4}, n_{5}, n_{6}\right): n_{i} \geqslant 0\right.$ for $1 \leqslant i \leqslant 6, n_{1}+n_{2} \geqslant n_{3}, n_{4}+n_{5} \geqslant$ $\left.n_{6}\right\}$.

Proof: (i) Recall that $\beta_{n}=\left(\operatorname{Ad}\left(W_{n} W_{n}^{\prime}\right) \circ R, A d\left(V_{n} V_{n}^{\prime}\right) \circ R\right)$ from which it follows that $B_{n}^{1}=\left\{f \in C\left(S^{1}, M_{q_{4 n}}\right): f(t)=W_{n} W_{n}^{\prime} f(\bar{t}) W_{n} W_{n}^{\prime}\right.$ for each $\left.t \in S^{1}\right\}$. Each element of $B_{n}^{1}$ is determined by its restriction to $\left\{t \in S^{1}: \operatorname{Im} t \geqslant 0\right\}$ and is therefore specified by the function $g: s \rightarrow f\left(e^{i \pi s}\right)$ on $[0,1]$ which satisfies $g(0), g(1) \in\left\{W_{n} W_{n}^{\prime}\right\}^{\prime}=\left\{E_{n}\right\}^{\prime}$. The result for $B_{n}^{2}$ is exactly similar.

(ii) By [10, Lemma 2.1], $K_{1}\left(B_{n}^{1}\right)$ is isomorphic to $K_{0}\left(M_{q_{4 n}}\right) /\left\{i_{*}(a)-i_{*}(b): a, b \in\right.$ $\left.K_{0}\left(\left\{E_{n}\right\}^{\prime}\right)\right\}$, where $i_{*}$ results from the inclusion map from $\left\{E_{n}\right\}^{\prime}$ into $M_{q_{4 n}}$. It is readily seen that $K_{0}\left(\left\{E_{n}\right\}^{\prime}\right)$ is isomorphic to $\mathbb{Z}^{2}$ with $i_{*}: \mathbb{Z}^{2} \rightarrow \mathbb{Z}$ given by $i_{*}\left(n_{1}, n_{2}\right)=$ $n_{1}+n_{2}$. Hence $K_{1}\left(B_{n}^{1}\right)$ is isomorphic to $\mathbb{Z} / \mathbb{Z}$. Similarly $K_{1}\left(B_{n}^{2}\right)$ is also zero, as therefore is $K_{1}\left(B_{n}\right)$.

(iii) By [10, Lemma 2.1], $K_{0}\left(B_{n}^{1}\right)$ is isomorphic to $\left\{(a, b): a, b \in K_{0}\left(\left\{E_{n}\right\}^{\prime}\right)\right.$, $\left.i_{*}(a)=i_{*}(b)\right\}$ which is isomorphic to $\left\{\left(n_{1}, n_{2}, m_{1}, m_{2}\right): n_{1}+n_{2}=m_{1}+m_{2}\right\}$, with positive cone given by the usual positive cone on $\mathbb{Z}^{4}$. Hence, restricting to the first three coordinates, $K_{0}\left(B_{n}^{1}\right)$ is isomorphic to $\mathbb{Z}^{3}$ with positive cone $\left\{\left(n_{1}, n_{2}, n_{3}\right): n_{1}, n_{2}, n_{3} \geqslant\right.$ $\left.0, n_{3} \leqslant n_{1}+n_{2}\right\}$. The result for $K_{0}\left(B_{n}^{2}\right)$ is exactly similar, from which the result for $K_{0}\left(B_{n}\right)$ follows.

The next step is to determine the $6 \times 6$ matrices corresponding to the maps $\theta_{n *}$ : $K_{0}\left(B_{n}\right) \rightarrow K_{0}\left(B_{n+1}\right)$ and to check that they are non-singular. In order to do this, an explicit expression for $E_{n}$ and $F_{n}$ will be obtained. The expression will involve matrices of the form $\left(I_{k}+Q_{k}\right) / 2$, where $Q_{k}$ was defined earlier: it will be convenient to denote such a matrix $\left(I_{k}+Q_{k}\right) / 2$ by $P_{k}$. 
LEMmA 2.1. For each $n \in \mathbb{N}$,
(i) $W_{n} W_{n}^{\prime}=\operatorname{diag}\left(Q_{k_{n}(1)}, Q_{k_{n}(2)}, \ldots, Q_{k_{n}\left(2^{n-1}\right)}\right)$
(ii) $V_{n} V_{n}^{\prime}=\operatorname{diag}\left(Q_{\ell_{n}(1)}, Q_{\ell_{n}(2)}, \ldots, Q_{\ell_{n}\left(2^{n-1}\right)}\right)$
(iii) $E_{n}=\operatorname{diag}\left(P_{k_{n}(1)}, P_{k_{n}(2)}, \ldots, P_{k_{n}\left(2^{n-1}\right)}\right)$
(iv) $F_{n}=\operatorname{diag}\left(P_{\ell_{n}(1)}, P_{\ell_{n}(2)}, \ldots, P_{\ell_{n}\left(2^{n-1}\right)}\right)$,

where the integers $k_{n}(i)$ and $\ell_{n}(i)$ are defined inductively by $k_{1}(1)=q_{4}, \ell_{1}(1)=q_{3}$ and, for each $n \in \mathbb{N}$ and each $1 \leqslant i \leqslant 2^{n-1}, k_{n+1}(i)=a_{n+1} k_{n}(i), k_{n+1}\left(2^{n-1}+i\right)=$ $b_{n+1} \ell_{n}(i), \ell_{n+1}(i)=c_{n+1} k_{n}(i)$ and $\ell_{n+1}\left(2^{n-1}+i\right)=d_{n+1} \ell_{n}(i)$.

PROOF: These are all straightforward consequences of the definitions, using the observation that, for any matrix $\left(x_{i j}\right)$, the matrix $\left(x_{i j}\right) \otimes Q_{k}$ consists of blocks $x_{i j} Q_{k}$. [

Lemma 2.1 can now be used to compute the images of the generators of $\mathbb{Z}^{6} \cong$ $K_{0}\left(B_{n}\right)$ under $\theta_{n *}$.

LEMMA 2, 2. Let $K_{0}\left(B_{n}\right)$ and $K_{0}\left(B_{n+1}\right)$ be identified with $\mathbb{Z}^{6}$ as in Proposition 2.1 and let $\alpha_{n+1}=\left[a_{n+1} / 2\right], \beta_{n+1}=\left[b_{n+1} / 2\right], \gamma_{n+1}=\left[c_{n+1} / 2\right]$ and $\delta_{n+1}=\left[d_{n+1} / 2\right]$, where $[x]$ denotes the integral part of $x$. Then

$$
\begin{aligned}
& \theta_{\theta *}(1,0,1,0,0,0)=\left(a_{n+1}-\alpha_{n+1}, \alpha_{n+1}, a_{n+1}-\alpha_{n+1}, c_{n+1}-\gamma_{n+1}, \gamma_{n+1}, c_{n+1}-\gamma_{n+1}\right) \\
& \theta_{n *}(0,1,0,0,0,0)=\left(\alpha_{n+1}, a_{n+1}-\alpha_{n+1}, \alpha_{n+1}, \gamma_{n+1}, c_{n+1}-\gamma_{n+1}, \gamma_{n+1}\right) \\
& \theta_{n *}(0,0,0,1,0,1)=\left(b_{n+1}-\beta_{n+1}, \beta_{n+1}, b_{n+1}-\beta_{n+1}, d_{n+1}-\delta_{n+1}, \delta_{n+1}, d_{n+1}-\delta_{n+1}\right) \\
& \theta_{n *}(0,0,0,0,1,0)=\left(\beta_{n+1}, b_{n+1}-\beta_{n+1}, \beta_{n+1}, \delta_{n+1}, d_{n+1}-\delta_{n+1}, \delta_{n+1}\right) .
\end{aligned}
$$

Proof: Define $e_{k_{n}(1)} \leqslant P_{k_{n}(1)}$ to be the $k_{n}(1) \times k_{n}(1)$ matrix whose sole non-zero entries are $1 / 2$ in each of the four corners and let $e=\operatorname{diag}\left(e_{k_{n}(1)}, 0_{k_{n}(2)}, \ldots, 0_{k_{n}\left(2^{n-1}\right)}\right)$, so that $e$ is a minimal projection in $E_{n} M_{q_{4 n}} E_{n}$ (regarded also as a constant element of $B_{n}^{1}$ ). Then

$$
\theta_{n}(e, 0)=\left(\left(\begin{array}{cc}
e \otimes I_{a_{n+1}} & 0 \\
0 & 0
\end{array}\right),\left(\begin{array}{cc}
e \otimes I_{c_{n+1}} & 0 \\
0 & 0
\end{array}\right)\right)
$$

Furthermore $P_{k_{n+1}(1)}\left(e \otimes I_{a_{n+1}}\right)=\left(I_{k_{n}(1)} \otimes I_{a_{n+1}}+Q_{k_{n}(1)} \otimes Q_{a_{n+1}}\right) / 2\left(e \otimes I_{a_{n+1}}\right)=$ $e \otimes P_{a_{n+1}}=\left(e \otimes I_{a_{n+1}}\right) P_{k_{n+1}(1)}$ and, similarly, $P_{\ell_{n+1}(1)}\left(e \otimes I_{c_{n+1}}\right)=e \otimes P_{c_{n+1}}=$ $\left(e \otimes I_{c_{n+1}}\right) P_{\ell_{n+1}(1)}$. Hence, using Lemma 2.1,

$$
\begin{aligned}
\left(E_{n+1}, 0\right) \theta_{n}(e, 0) & =\left(\operatorname{diag}\left(e \otimes P_{a_{n+1}}, 0\right), 0\right) \\
& =\theta_{n}(e, 0)\left(E_{n+1}, 0\right)
\end{aligned}
$$

and

$$
\begin{aligned}
\left(0, F_{n+1}\right) \theta_{n}(e, 0) & =\left(0, \operatorname{diag}\left(e \otimes P_{c_{n+1}}, 0\right)\right) \\
& =\theta_{n}(e, 0)\left(0, F_{n+1}\right) .
\end{aligned}
$$


It follows that $\theta_{n *}(1,0,1,0,0,0)=\left(\tau_{1}, \tau_{2}, \tau_{1}, \sigma_{1}, \sigma_{2}, \sigma_{1}\right)$ where $\tau_{1}=\operatorname{Tr}\left(e \otimes P_{a_{n+1}}\right), \tau_{2}=$ $\operatorname{Tr}\left(e \otimes I_{a_{n+1}}\right)-\tau_{1}=a_{n+1}-\tau_{1}, \sigma_{1}=\operatorname{Tr}\left(e \otimes P_{c_{n+1}}\right)$ and $\sigma_{2}=\operatorname{Tr}\left(e \otimes I_{c_{n+1}}\right)-\sigma_{1}=$ $c_{n+1}-\sigma_{1}$. However $\operatorname{Tr}\left(e \otimes P_{a_{n+1}}\right)=\operatorname{Tr}\left(P_{a_{n+1}}\right)=\left[\left(a_{n+1}+1\right) / 2\right]=a_{n+1}-$ $\left[a_{n+1} / 2\right]$ and, similarly, $\operatorname{Tr}\left(e \otimes P_{c_{n+1}}\right)=c_{n+1}-\left[c_{n+1} / 2\right]$. Hence $\theta_{n *}(1,0,1,0,0,0)=$ $\left(a_{n+1}-\alpha_{n+1}, \alpha_{n+1}, a_{n+1}-\alpha_{n+1}, c_{n+1}-\gamma_{n+1}, \gamma_{n+1}, c_{n+1}-\gamma_{n+1}\right)$. The image of $(0,0,0,1,0,1)$ is computed in exactly the same way using $\theta_{n}(0, e)$ where $e$ is a minimal projection in $F_{n} M_{q_{4 n-1}} F_{n}$ obtained from $e_{\ell_{n}(1)} \leqslant P_{\ell_{n}(1)}$. The other two images are then computed in a similar manner but based on minimal projections

$$
e_{k_{n}(1)}=\frac{1}{2}\left(\begin{array}{cc}
1 & -1 \\
-1 & 1
\end{array}\right) \leqslant 1-P_{k_{n}(1)}
$$

and

$$
e_{\ell_{n}(1)}=\frac{1}{2}\left(\begin{array}{cc}
1 & -1 \\
-1 & 1
\end{array}\right) \leqslant 1-P_{\ell_{n}(1)} .
$$

In order to find the images of other generators, such as $(1,0,0,0,0,0)$ and $(0,0,0,1,0,0)$, it is necessary to consider non-constant projections and to find their image under $\theta_{n}$. This is done in the following lemma.

LEMma 2.3. Maintaining the notation of Lemma 2.2,

$$
\begin{aligned}
& \theta_{n *}(1,0,0,0,0,0) \\
& \quad=\left(\alpha_{n+1}+1, a_{n+1}-\alpha_{n+1}-1, \alpha_{n+1}, \gamma_{n+1}+1, c_{n+1}-\gamma_{n+1}-1, \gamma_{n+1}+1\right)
\end{aligned}
$$

and

$$
\begin{aligned}
& \theta_{n *}(0,0,0,1,0,0) \\
& \quad=\left(\beta_{n+1}+1, b_{n+1}-\beta_{n+1}-1, \beta_{n+1}+1, \delta_{n+1}+1, d_{n+1}-\delta_{n+1}-1, \delta_{n+1}\right) .
\end{aligned}
$$

PROOF: As in the proof of Lemma 2.2, construct a minimal projection $e$ with $e(0)$ in $E_{n} M_{q_{4 n}} E_{n}$ and $e(1)$ in $\left(1-E_{n}\right) M_{q_{4 n}}\left(1-E_{n}\right)$, starting with a minimal $k_{n}(1) \times k_{n}(1)$ projection $e_{k_{n}(1)}$ defined by

$$
e_{k_{n}(1)}(s)=\frac{1}{2}\left(\begin{array}{cc}
1 & e^{\pi i s} \\
e^{-\pi i s} & \\
1
\end{array}\right)
$$

which satisfies $e_{k_{n}(1)}(0) \leqslant P_{k_{n}(1)}$ and $e_{k_{n}(1)}(1) \leqslant 1-P_{k_{n}(1)}$. Then, using Lemma 2.1, $e(0) \leqslant E_{n}$ and $e(1) \leqslant 1-E_{n}$, so $[e] \in K_{0}\left(B_{n}^{1}\right)$ corresponds to $(1,0,0) \in \mathbb{Z}^{3}$. In 
order to calculate $\theta_{n}(e, 0)$ it is convenient to regard $e$ as an element of $C\left(S^{1}, M_{q_{4 n}}\right)$ by identifying $e_{k_{n}(1)}$ above with the matrix valued function defined on $S^{1}$ by

$$
e_{k_{n}(1)}(t)=\frac{1}{2}\left(\begin{array}{ll}
1 & t \\
\bar{t} & 1
\end{array}\right)
$$

Note that $Q_{k_{n}(1)} e_{k_{n}(1)}(t) Q_{k_{n}(1)}=e_{k_{n}(1)}(\bar{t})$ so, using Lemma 2.1, $W_{n} W_{n}^{\prime} e(t) W_{n} W_{n}^{\prime}=$ $e(\bar{t})$, as required for $e$ to belong to the fixed point algebra $B_{n}^{1}$ of $\beta_{n}$ restricted to $A_{n}^{1}$.

The image of $\theta_{n}(e, 0)$ can be found by writing $e_{k_{n}(1)}$ as

$$
e_{k_{n}(1)}=\frac{1}{2}\left(\begin{array}{cc}
1 & \\
& 1
\end{array}\right)+\frac{1}{2} \mathrm{id}\left(\begin{array}{l}
1 \\
\end{array}\right)+\frac{1}{2} \mathrm{id}^{*}\left(\begin{array}{l} 
\\
1
\end{array}\right)
$$

from which it follows that $\theta_{n}(e, 0)=(\operatorname{diag}(f, 0), \operatorname{diag}(g, 0))$ where $f$ is the $k_{n+1}(1) \times$ $k_{n+1}(1)$ matrix and $g$ is the $\ell_{n+1}(1) \times \ell_{n+1}(1)$ matrix given by

$$
\begin{aligned}
f & =\frac{1}{2}\left(\begin{array}{ll}
I_{a_{n+1}} & \\
& I_{a_{n+1}}
\end{array}\right)+\frac{1}{2}\left(\begin{array}{l}
1 \\
a_{a_{n+1}}
\end{array}\right) \otimes R_{a_{n+1}}+\frac{1}{2}\left(\begin{array}{l}
1 \\
1
\end{array}\right) \otimes R_{a_{n+1}}^{*} \\
& =\frac{1}{2}\left(\begin{array}{cc}
I_{a_{n+1}} \\
R_{a_{n+1}}^{*} & I_{a_{n+1}}
\end{array}\right)
\end{aligned}
$$

and, similarly,

$$
g=\frac{1}{2}\left(\begin{array}{ll}
I_{c_{n+1}} & S_{c_{n+1}} \\
S_{c_{n+1}}^{*} & I_{c_{n+1}}
\end{array}\right) .
$$

To calculate $\left(E_{n+1}, 0\right) \theta_{n}(e, 0)(1)$ observe that

$$
\begin{aligned}
P_{k_{n+1}(1)} f(1) & =\frac{1}{4}\left(\begin{array}{ccc}
I_{a_{n+1}} & & Q_{a_{n+1}} \\
& \ddots \ddots & \\
Q_{a_{n+1}} & & I_{a_{n+1}}
\end{array}\right)\left(\begin{array}{cc}
I_{a_{n+1}} & R_{a_{n+1}}(1) \\
R_{a_{n+1}}^{*}(1) & I_{a_{n+1}}
\end{array}\right) \\
& =\frac{1}{4}\left(\begin{array}{cc}
I_{a_{n+1}}+Q_{a_{n+1}} R_{a_{n+1}}^{*}(1) & R_{a_{n+1}}(1)+Q_{a_{n+1}} \\
R_{a_{n+1}}^{*}(1)+Q_{a_{n+1}} & I_{a_{n+1}}+Q_{a_{n+1}} R_{a_{n+1}}(1)
\end{array}\right)
\end{aligned}
$$

From $Q_{a_{n+1}} R_{a_{n+1}}^{*}(t)=R_{a_{n+1}}(\bar{t}) Q_{a_{n+1}}$ it follows that $P_{k_{n+1}(1)} f(1)=f(1) P_{k_{n+1}(1)}$ and therefore that $\left(E_{n+1}, 0\right) \theta_{n}(e, 0)(1)=\theta_{n}(e, 0)(1)\left(E_{n+1}, 0\right)$. The trace is given by $\operatorname{Tr}\left(E_{n+1} \operatorname{diag}(f(1), 0)\right)=\operatorname{Tr}\left(P_{k_{n+1}(1)} f(1)\right)=\left[a_{n+1} / 2\right]+1$ (as can be seen by a separate analysis of the cases $a_{n+1}$ odd and $a_{n+1}$ even). Similarly $\left(E_{n+1}, 0\right) \theta_{n}(e, 0)(-1)=$ 
$\theta_{n}(e, 0)(-1)\left(E_{n+1}, 0\right)$ with $\operatorname{Tr}\left(E_{n+1} \operatorname{diag}(f(-1), 0)\right)=\operatorname{Tr}\left(P_{k_{n+1}(1)} f(-1)\right)=\left[a_{n+1} / 2\right]$. Thus the first three components of $\theta_{n *}(1,0,0,0,0,0)$ are $\alpha_{n+1}+1, a_{n+1}-\alpha_{n+1}-1, \alpha_{n+1}$. The second three components are calculated similarly considering $\left(0, F_{n+1}\right) \theta_{n}(e, 0)$ via $P_{\ell_{n+1}(1)} g$ where $g$ is constant. The formula for $\theta_{n *}(0,0,0,1,0,0)$ then follows by an exactly similar argument.

Proposition 2.2. Let $A_{n}$ be the $6 \times 6$ matrix corresponding to $\theta_{n *}$ : $K_{0}\left(B_{n}\right) \rightarrow K_{0}\left(B_{n+1}\right)$. Then $\operatorname{det}\left(A_{n}\right)= \pm 1$.

Proof: To ease the complexity of the notation omit subscripts so, by Lemmas 2.2 and 2.3,

$$
A_{n}=\left(\begin{array}{cccccc}
\alpha+1 & \alpha & a-2 \alpha-1 & \beta+1 & \beta & b-2 \beta-1 \\
a-\alpha-1 & a-\alpha & 2 \alpha+1-a & b-\beta-1 & b-\beta & 2 \beta+1-b \\
\alpha & \alpha & a-2 \alpha & \beta+1 & \beta & b-2 \beta-1 \\
\gamma+1 & \gamma & c-2 \gamma-1 & \delta+1 & \delta & d-2 \delta-1 \\
c-\gamma-1 & c-\gamma & 2 \gamma+1-c & d-\delta-1 & d-\delta & 2 \delta+1-d \\
\gamma+1 & \gamma & c-2 \gamma-1 & \delta & \delta & d-2 \delta
\end{array}\right)
$$

Denoting the $i$ th column by $C_{i}$ and the $i$ th row by $R_{i}$ and performing the following sequence of row and column operations:

$$
\begin{aligned}
& C_{1}^{\prime}=C_{1}-C_{2}, C_{4}^{\prime}=C_{4}-C_{5}, C_{3}^{\prime}=C_{3}+C_{2}, C_{6}^{\prime}=C_{6}+C_{5} \\
& R_{2}^{\prime}=R_{2}+R_{1}, R_{5}^{\prime}=R_{4}+R_{5}, R_{1}^{\prime}=R_{1}-R_{3}, R_{4}^{\prime}=R_{4}-R_{6} \\
& C_{3}^{\prime}=C_{3}+C_{2}+C_{1}, C_{6}^{\prime}=C_{6}+C_{5}+C_{4}
\end{aligned}
$$

yields

$$
\left(\begin{array}{cccccc}
1 & 0 & 0 & 0 & 0 & 0 \\
0 & a & 2 a & 0 & b & 2 b \\
0 & \alpha & a & 1 & \beta & b \\
0 & 0 & 0 & 1 & 0 & 0 \\
0 & c & 2 c & 0 & d & 2 d \\
1 & \gamma & c & 0 & \delta & d
\end{array}\right)
$$

Using the fact that $a d-b c=1$, the determinant of this matrix reduces to $(a-2 \alpha)$ $(d-2 \delta)+(c-2 \gamma)(2 \beta-b)$. Thus when either $a$ or $d$ is even (so that both $b$ and $c$ are odd) $\operatorname{det}\left(A_{n}\right)=0-1=-1$ and when either $b$ or $c$ is even (so that both $a$ and $d$ are odd) $\operatorname{det}\left(A_{n}\right)=1-0=1$. Since $a d-b c=1$ these are the only possible cases.

Proposition 2.3. Let $B$ be the fixed point algebra of $A_{\theta}$ under the automorphism $\beta$. Then $K_{0}(B)$ is isomorphic to $\mathbb{Z}^{6}$ and $K_{1}(B)$ is isomorphic to $\{0\}$.

Proof: $B$ is the inductive limit of the sequence $B_{1} \stackrel{\theta_{1}}{\longrightarrow} B_{2} \stackrel{\theta_{2}}{\longrightarrow} \ldots$, so the result follows immediately from Propositions 2.1 and 2.2. 
Proposition 2.3 is the basis for showing that $B$ is isomorphic to the fixed point algebra $B_{\theta}$ of the flip. Another key ingredient is the observation that $B$ is simple with a unique tracial state.

Proposition 2.4. Let $B$ be the fixed point subalgebra of $A_{\theta}$ under the automorphism $\beta$. Then

(a) $B$ is simple;

(b) $B$ has a unique tracial state (obtained by restricting that on $A_{\theta}$ ).

ProOF: (a) The action of $\beta$ on $K_{1}\left(A_{\theta}\right)$ is given by $-I$ whereas any unitarily implemented automorphism acts trivially on $K_{1}\left(A_{\theta}\right)$. Hence by a standard result, described in [12, Theorem 8.10.12], the fixed point algebra $B$ of $\beta$ is simple.

(b) It is shown in [3, Remark 4.7] that it is sufficient to show that the crossed product $M_{\theta} \times_{\bar{\beta}} \mathbb{Z}_{2}$ of the weak closure $M_{\theta}$ of $A_{\theta}$ in the trace representation by the unique extension $\bar{\beta}$ of $\beta$ to $M_{\theta}$ is a factor. Equivalently, it is sufficient to show that the weak closure of $B$ in the trace representation is a factor, that is, that the unique trace on $A_{\theta}$ restricts to a factor state on $B$. Thus the tracial state space of $B$ will now be investigated.

The commutative diagram

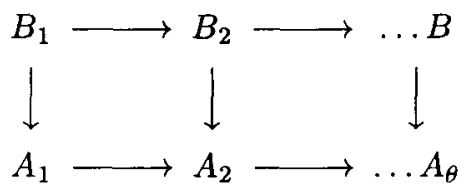

where the vertical maps are inclusions, gives rise to a commutative diagram of tracial state spaces

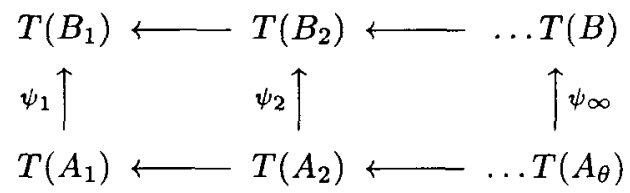

Recall that $A_{n}=M_{q_{4 n}}\left(C\left(S^{1}\right)\right) \oplus M_{q_{4 n-1}}\left(C\left(S^{1}\right)\right)$ from which it follows that $T\left(A_{n}\right)$ has a direct sum decomposition $T_{A_{n}}^{1} \oplus T_{A_{n}}^{2}$. For each probability measure $\mu$ on $S^{1}$ there exists a trace $\tau_{\mu}^{q}$ on $M_{q}\left(C\left(S^{1}\right)\right)$ defined by $\tau_{\mu}^{q}(f)=\int \operatorname{Tr}(f) d \mu$, where $\operatorname{Tr}$ is the normalised trace on $M_{q}$, and $T_{A_{n}}^{i}=\left\{\tau_{\mu}^{q}: \mu \in M_{1}^{+}\left(S^{1}\right)\right\}$ for the appropriate choice of $q$ that is, $q_{4 n}$ for $T_{A_{n}}^{1}$ or $q_{4 n-1}$ for $T_{A_{n}}^{2}$. Similarly $T\left(B_{n}\right)$ has a direct sum decomposition $T_{B_{n}}^{1} \oplus T_{B_{n}}^{2}$, where $T_{B_{n}}^{1}$ is the tracial state space of $\left\{f \in C\left([0,1], M_{q}\right): f(0), f(1) \in\right.$ $\left.\left\{E_{n}\right\}^{\prime}\right\}$. Using $M_{1}^{+}(0,1)$ to denote the set of probability measures $\mu$ on $[0,1]$ for which $\mu(\{0\})=\mu(\{1\})=0$ and $\tau_{\mu}$ to denote the associated trace in $T_{B_{n}}^{1}$, the trace space $T_{B_{n}}^{1}$ is the convex hull of the face $F_{n}^{1}=\left\{\tau_{\mu}: \mu \in M_{1}^{+}(0,1)\right\}$ and four other traces 
$\tau_{0, E}, \tau_{0,1-E}, \tau_{1, E}, \tau_{1,1-E}$. The first of these is defined by $\tau_{0, E}(f)=\operatorname{Tr}\left(E_{n} f(0)\right)$, where Tr is the normalised trace on $E_{n} M_{q_{4 n}} E_{n}$, and the others are defined similarly.

Let $K_{n}^{1}$ be $\left\{\tau_{\mu}: \mu \in M_{1}^{+}[0,1]\right\}$, which is equal to the convex hull of $F_{n}^{1}, \lambda \tau_{0, E}+$ $(1-\lambda) \tau_{0,1-E}$ and $\lambda \tau_{1, E}+(1-\lambda) \tau_{1,1-E}$, where $\lambda=\operatorname{dim}\left(E_{n}\right) /\left(q_{4 n}-\operatorname{dim}\left(E_{n}\right)\right)$, and let $K_{n}^{2}$ be defined similarly. The image of $T\left(A_{n}\right)$ under the mapping $\psi_{n}$ is $K_{n}^{1} \oplus K_{n}^{2}$ and it follows from the uniqueness of the trace on $A_{\theta}$ that there is therefore a unique element in the limit $K$ of the system

$$
K_{1}^{1} \oplus K_{1}^{2} \leftarrow K_{2}^{1} \oplus K_{2}^{2} \leftarrow \ldots
$$

This unique element $\tau_{\lambda}$ corresponds to the restriction to $B$ of the unique trace on $A_{\theta}$. It belongs to the face $F$ of $T(B)$ determined by the system of faces

$$
F_{1}^{1} \oplus F_{1}^{2} \leftarrow F_{2}^{1} \oplus F_{2}^{2} \leftarrow \ldots
$$

and therefore this face $F$, which is contained in $K$, contains a unique element. Hence $\tau_{\lambda}$ is an extreme point of $T(B)$ and so, by $[7,6.8 .6$ and 6.8.7], is a factor trace, as required.

Proposition 2.5. $B$ is a simple $A F$ algebra.

Proof: By Proposition $2.4 B$ is a simple unital $C^{*}$-algeba with a unique tracial state and, by construction, $B$ is an inductive limit of the algebras $B_{n}=B_{n}^{1} \oplus B_{n}^{2}$. Hence (as observed in [16, Theorem 3.0]) $B$ is covered by the classification results of $\mathrm{Su}$, obtained in [13] and [14], from which the result follows.

Propositions 2.4 and 2.5 can now be used to determine the order structure on $K_{0}(B)$ and hence to compare it with the known order structure on $K_{0}\left(B_{\theta}\right)$.

PROPOSITION 2.6. There is an order isomorphism from the group $K_{0}(B)$ onto $K_{0}\left(B_{\theta}\right)$.

Proof: The ordered group $K_{0}\left(B_{\theta}\right)$ is isomorphic to the ordered group $K_{0}\left(C_{\theta}\right)$, where $C_{\theta}$ is the crossed product of $A_{\theta}$ by the flip. However $K_{0}\left(C_{\theta}\right)$ has been computed by Kumjian [11] to be equal to $\mathbb{Z}^{6}$. Its order structure has been investigated in [15] where it is shown (in the course of the proof of [15, Theorem 4.1]) that there are generators for $\mathbb{Z}^{6}$ such that the coordinate 6-tuple $\left(n_{1}, n_{2}, n_{3}, n_{4}, n_{5}, n_{6}\right)$ is positive if and only if it is zero or $2 n_{1}+n_{2}+n_{3}+n_{4}+n_{5}+\theta n_{6}>0$. Let $A$ be the invertible $6 \times 6$ matrix

$$
A=\left(\begin{array}{cccccc}
0 & 1 & 0 & 0 & 0 & 0 \\
0 & 0 & 1 & 0 & 0 & 0 \\
0 & 0 & 0 & 1 & 0 & 0 \\
0 & 0 & 0 & 0 & 0 & 1 \\
0 & -2 & -1 & 0 & 1 & -1 \\
1 & 1 & 0 & 0 & 0 & 0
\end{array}\right)
$$


and note that if $A x=y$ then $2 y_{1}+y_{2}+y_{3}+y_{4}+y_{5}+\theta y_{6}=\theta x_{1}+\theta x_{2}+x_{4}+$ $x_{5}$, so that $K_{0}\left(B_{\theta}\right)$ is isomorphic to $\mathbb{Z}^{6}$ with positive cone consisting of 0 and $\left\{\left(n_{1}, n_{2}, n_{3}, n_{4}, n_{5}, n_{6}\right): \theta n_{1}+\theta n_{2}+n_{4}+n_{5}>0\right\}$. However, using [2, Propositions 2.4 and 2.5 and Theorem 3.1], the ordering on $K_{0}(B)$ is the strict ordering determined by the unique trace $\tau$.

Let $p_{n}: K_{0}\left(B_{n}\right) \rightarrow K_{0}\left(A_{n}\right)$ be the map arising from the inclusion $B_{n} \rightarrow A_{n}$ and let $p_{\infty}$ be the corresponding map from $K_{0}(B)$ to $K_{0}(A)$. Then $\tau_{*}\left(n_{1}, \ldots, n_{6}\right)=$ $\tau_{*} p_{\infty}\left(n_{1}, \ldots, n_{6}\right)$, where $\tau$ is used both for the unique trace on $A_{\theta}$ and its restriction to $B$. However it is known that $\tau_{*}\left(m_{1}, m_{2}\right)=\theta m_{1}+m_{2}$ for $\left(m_{1}, m_{2}\right) \in \mathbb{Z}^{2} \cong K_{0}\left(A_{\theta}\right)$ and so the proof will be complete if it is shown that $p_{\infty}\left(n_{1}, n_{2}, n_{3}, n_{4}, n_{5}, n_{6}\right)=$ $\left(n_{1}+n_{2}, n_{4}+n_{5}\right)$. It is clear from a consideration of the trace of constant projections that, for each $n \in \mathbb{N}, p_{n}\left(n_{1}, n_{2}, n_{3}, n_{4}, n_{5}, n_{6}\right)=\left(n_{1}+n_{2}, n_{4}+n_{5}\right)$. So consider the following commutative diagram

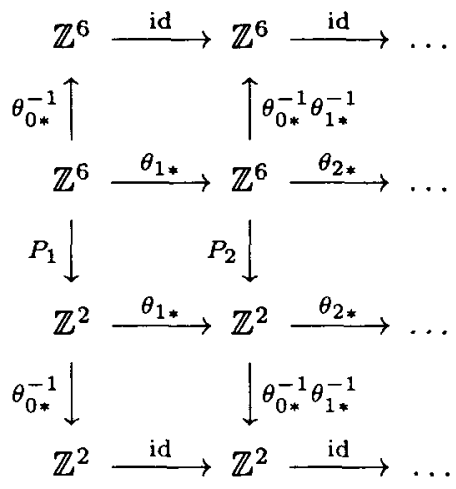

where $\theta_{0 *}$ is defined on $\mathbb{Z}^{6}$ by a matrix $A_{0}$ as in Proposition 2.5 with $a_{0}=q_{4}, b_{0}=$ $p_{4}, c_{0}=q_{3}, d_{0}=p_{3}$ and is defined on $\mathbb{Z}^{2}$ by the $2 \times 2$ matrix with entries $a_{0}, b_{0}, c_{0}, d_{0}$. It follows that $p_{\infty}\left(n_{1}, n_{2}, n_{3}, n_{4}, n_{5}, n_{6}\right)=\theta_{0 *}^{-1} p_{1} \theta_{0 *}\left(n_{1}, n_{2}, n_{3}, n_{4}, n_{5}, n_{6}\right)=\left(n_{1}+n_{2}\right.$, $\left.n_{4}+n_{5}\right)$, as required.

The major result of this section can now be obtained:

THEOREM 2.1. Let $\beta$ be the isomorphim from $\mathbb{Z}_{2} \times \mathbb{Z}_{2}$ to Aut $\left(A_{\theta}\right)$ described in Theorem 1.1. Then the fixed point algebra $B$ of $\beta(1,1)$ is isomorphic to the fixed point algebra $B_{\theta}$ of the flip determined by $U \mapsto U^{*}$ and $V \mapsto V^{*}$.

Proof: By [5] $B_{\theta}$ is an AF algebra so, by Proposition 2.5 and the classification theorem for AF algebras, it suffices to show that the order isomorphism of Proposition 2.6 preserves the scales. From the commutative diagram in the proof of Proposition 2.6, the order unit of $K_{0}(B)$ corresponding to the identity of $B$ is $\theta_{0 *}^{-1}\left(d_{4}, q_{4}-d_{4}, d_{4}, d_{3}, q_{3}-d_{3}, d_{3}\right)=(0,0,0,0,1,0)$ where $d_{4}=\left[q_{4} / 2\right]$ and $d_{3}=\left[q_{3} / 2\right]$. If $A$ is the $6 \times 6$ matrix used in the proof of Proposition 2.6 then $A^{-1}(0,0,0,0,1,0)^{t r}=$ 
$(0,0,0,0,1,0)^{t r}$ so, by $\left[1\right.$, Theorem 7.4.3], it suffices to show that $\tau_{*}^{B}(0,0,0,0,1,0)=1$, where $\tau^{B}$ is the normalised trace on $B_{\theta}$. However it is shown in the course of the proof of $\left[15\right.$, Theorem 4.1] that $\tau_{*}^{C}(0,0,0,0,1,0)=1 / 2$, where $\tau^{C}$ is the normalised trace on the crossed product $C_{\theta}=A_{\theta} \times \mathbb{Z}_{2}$ of $A_{\theta}$ by the flip. However when $B_{\theta}$ is embedded in $C_{\theta}$ in the usual way, described for example in [4, Section 4], $\tau^{B}=2 \tau^{C}$. Hence $\tau_{*}^{B}(0,0,0,0,1,0)=1$, as required.

\section{REFERENCES}

[1] B. Blackadar, K-theory for operator algebras (Springer-Verlag, Berlin, Heidelberg, New York, 1986).

[2] B. Blackadar, 'Traces on simple AF C*-algebras', J. Funct. Anal. 38 (1980), 156-168.

[3] O. Bratteli, G.A. Elliott, D.E. Evans and A. Kishimoto, 'Non-commutative spheres I', Internat. J. Math. 2 (1991), 139-166.

[4] O. Bratteli, G.A. Elliott, D.E. Evans and A. Kishimoto, 'Non-commutative spheres II: rational rotations', $J$. Operator Theory 27 (1992), 53-85.

[5] O. Bratteli and A. Kishimoto, 'Non-commutative spheres III: irrational rotations', Comm. Math. Phys, 147 (1992), 605-624.

[6] B. Brenken, 'Representations and automorphisms of the irrational rotation algebra', $\mathrm{Pa}$ cific J. Math. 111 (1984), 257-282.

[7] J. Dixmier, Les $C^{*}$-algèbres et leurs représentations (Gauthier-Villars, Paris, 1964).

[8] G.A. Elliott, 'On the classification of $C^{*}$-algebras of real rank zero', J. Reine Angew. Math. 443 (1993), 179-219.

[9] G.A. Elliott and D.E. Evans, 'The structure of the irrational rotation $C^{*}$-algebra', Ann. of Math. 138 (1993), 477-501.

[10] D.E. Evans and A. Kishimoto, 'Compact group actions on UHF algebras obtained by folding the interval', J. Funct. Anal. 98 (1991), 346-360.

[11] A. Kumjian, 'On the $K$-theory of the symmetrized non-commutative torus', C.R. Math. Rep. Acad. Sci. Canada 12 (1990), 87-89.

[12] G.K. Pedersen, $C^{*}$-algebras and their automorphism groups (Academic Press, London, 1979).

[13] H. Su, 'On the classification of $C^{*}$-algebras of real rank zero: inductive limits of matrix algebras over graphs', C.R. Math. Rep. Acad. Sci. Canada 13 (1991), 223-228.

[14] H. Su, 'On the classification of $C^{*}$-algebras of real rank zero: inductive limits of matrix algebras over non-Hausdorff graphs', Mem. Amer. Math. Soc. 547 (1995).

[15] S.G. Walters, 'Projective modules over the non-commutative sphere', J. London Math. Soc. 51 (1995), 589-602.

[16] S.G. Walters, 'Inductive limit automorphisms of the irrational rotation algebra', Comm. Math. Phys. 171 (1995), 365-381.

[17] Y. Watatani, 'Toral automorphisms on irrational rotation algebras', Math. Japon 26 (1981), 479-484. 
School of Mathematics

La Trobe University

Bundoora Vic 3083

Australia

e-mail: P.Stacey@latrobe.edu.au 